\title{
Bifurcations in One Degree-of-Vibration Quantum Billiards
}

\author{
Mason A. Porter and Richard L. Liboff \\ Center for Applied Mathematics \\ and \\ Schools of Electrical Engineering and Applied Physics \\ Cornell University
}

April, 2000

\begin{abstract}
We classify the local bifurcations of one dov quantum billiards, showing that only saddle-center bifurcations can occur. We analyze the resulting planar system when there is no coupling in the superposition state. In so doing, we also consider the global bifurcation structure. Using a double-well potential as a representative example, we demonstrate how to locate bifurcations in parameter space. We also discuss how to approximate the cuspidal
\end{abstract}


loop using AUTO as well as how to cross it via continuation by detuning the dynamical system. Moreover, we show that when there is coupling, the resulting five-dimensional system - though chaotic - has a similar underlying structure. We verify numerically that both homoclinic orbits and cusps occur and provide an outline of an analytical argument for the existence of such homoclinic orbits. Small perturbations of the system reveal homoclinic tangles that typify chaotic behavior.

\section{MSC NOS 37N20, 37K55, 37M20}

\section{Introduction}

Quantum chaos is an underdeveloped area of dynamical systems theory. One purpose of studying it is to generalize the notions of classical Hamiltonian chaos to the quantum regime, which has not yet been done in a universally accepted manner. One type of quantum chaos is often called semiquantum chaos, as these systems consist of classical (Hamiltonian) variables coupled with quantum variables. [4] Vibrating quantum billiards are a representative example of semiquantum chaotic systems. [17, 21] They may be used as models for quantum well microdevice components (such as quantum dots and quantum wires), Fermi accelerators [2], and intranuclear particle behavior.

In the present paper, we consider the bifurcation structure of vibrating billiard systems. Quantum billiards describe the motion of a point particle undergoing perfectly elastic collisions in a bounded domain. The particle's motion is described by the Schrödinger equation with Dirichlet boundary 
conditions. One defines the "degree-of-vibration" (dov) of a billiard as the number of boundary dimensions that vary with time. If the boundary is time-independent, the billiard is said to have zero dov. The linear vibrating billiard and the radially vibrating spherical billiard have a single dov, and the rectanglular billiard with time-varying length and width has two dov.

A zero dov quantum billiard exhibits only integrable behavior if it is globally separable. [13] It must, for example, be convex and describable by a non-composite geometry. [15, 19] If part of the billiard is concave (or is composite, like the stadium billiard), it may behave chaotically, as it shares many of the instability properties of the Anosov diffeomorphism. Blümel and Esser [3] found quantum chaos in the one-dimensional vibrating quantum billiard. Liboff and Porter [17] extended these results to spherical quantum billiards with vibrating surfaces and derived necessary conditions for chaotic behavior. They also generalized their results to other one dov billiards. 211 The purpose of the present paper is to examine bifurcations in single dov quantum billiards that occur when one alters the potential in which the billiard resides.

Vibrating quantum billiards are important for several reasons. Though an idealized model, they are nevertheless useful for the study of quantum chaos. From a more practical standpoint, vibrating quantum billiards may be applied to several problems in physics. The radially vibrating spherical quantum billiard, for example, may be used as a model for particle behavior in the nucleus [24] as well as for the quantum dot microdevice component. [18]. Additionally, the vibrating cylindrical billiard may be used as a model for the quantum wire, another microdevice component. 255 Other geometries of 
vibrating quantum billiards have similar applications. They may also be used as models of Fermi acceleration of cosmic rays. [2] The study of quantum chaos in vibrating billiard systems is thus important both because it expands the mathematical theory of dynamical systems and because it can be applied to problems in nuclear and mesoscopic physics.

In the present paper, we show that saddle-centers are the only type of bifurcations that can occur in one dov quantum billiards. When there is no coupling in the superposition state, we show how to analyze the resulting planar system analytically and numerically. Considering a double-well potential as a representative example, we demonstrate how to locate bifurcations in parameter space. We also discuss how to approximate the cuspidal loop using AUTO as well as how to continue past it by detuning the dynamical system. We also mention a shooting method for a more detailed analysis of the cuspidal loop. Moreover, we show that when there is coupling, the resulting five-dimensional system - though chaotic - has a similar underlying structure. We verify numerically that both homoclinic orbits and cusps occur, and we outline an analytic argument that demonstrates the existence of homoclinic orbits. Small perturbations of the system reveal homoclinic tangles that typify chaotic behavior.

\section{Equations of Motion}

The goal of the present project is to examine the behavior of one dov quantum billiards in various potentials in order to determine the effects of the potential on the behavior of the system. In particular, we analyze bifurca- 
tions of equilibria both analytically and numerically. We consider a two-state superposition solution to the vibrating billiard, and we examine the above problem for both the case in which the two states experience coupling and that in which they do not.

The present problem is described by the Schrödinger equation with solutions that are constrained to vanish on a time-dependent boundary $a(t)$. 21 That is,

$$
\psi(r, t ; a(t))=0 \text { for } r=a(t) .
$$

Because of the time-dependence of the boundary, the above boundary condition is nonlinear. The (mathematical) problem at hand is to find a boundary $a(t)$ such that Dirichlet boundary conditions are satisfied on it. One can then, in principle, insert $a(t)$ into the eigenfunctions in our normal mode expansion of the wave $\psi(r, t ; a(t))$ in order to obtain nonlinear normal modes. When the radius $a(t)$ behaves chaotically, the nonlinear normal modes are examples of quantum-mechanical wave chaos. One derives coupled nonlinear ordinary differential equations for $a(t)$ (and other variables) using a Galërkin approximation. [11, 22 Considering a two-term superposition state then corresponds to taking a two-term Galërkin projection. The equations of motion that one obtains depend on whether a particular subset of the quantum numbers in the two states are the same. 21] For the case of the radially vibrating sphere, the quantum numbers in question are the orbital and azimuthal quantum numbers $l$ and $m$, respectively. [17]

If these quantum numbers are the same in the two states, there is a 
coupling between them. The evolution of the system is then described by

$$
\begin{gathered}
\dot{x}=-\frac{\omega_{0} y}{a^{2}}-\frac{2 \mu P z}{M a}, \\
\dot{y}=\frac{\omega_{0} x}{a^{2}}, \\
\dot{z}=\frac{2 \mu P x}{M a}, \\
\dot{a}=\frac{P}{M},
\end{gathered}
$$

and

$$
\dot{P}=-\frac{\partial V}{\partial a}+\frac{2\left[\epsilon_{+}+\epsilon_{-}(z-\mu x)\right]}{a^{3}}
$$

where $x, y$, and $z$ are Bloch variables [1]

$$
\begin{gathered}
x=\rho_{12}+\rho_{21}, y=i\left(\rho_{21}-\rho_{12}\right), z=\rho_{22}-\rho_{11}, \\
x^{2}+y^{2}+z^{2}=1,
\end{gathered}
$$

$\rho_{m n} \equiv A_{m} A_{n}^{*}$ is the density matrix [16], $a$ is a displacement, $P$ is its conjugate momentum, $M$ is the mass of the billiard, $m \ll M$ is the mass of the confined particle, $\mu>0$ is the coupling coefficient between the two eigenstates, $V=$ $V(a)$ is the potential of the billiard boundary, $\omega_{0} \equiv \frac{\epsilon_{2}-\epsilon_{1}}{\hbar}, \epsilon_{ \pm} \equiv \frac{\left(\epsilon_{2} \pm \epsilon_{1}\right)}{2}$, and $\epsilon_{1}$ and $\epsilon_{2}\left(\epsilon_{2} \geq \epsilon_{1}\right)$ are the energies of the two states. It has been shown that these equations exhibit quantum chaotic behavior. 21]

If there is no coupling between the two eigenstates, the evolution of the system is described by a one degree-of-freedom Hamiltonian. The equations 
of motion are

$$
\begin{gathered}
\dot{a}=\frac{P}{M}, \\
\dot{P}=-\frac{\partial V}{\partial a}+\frac{\lambda}{a^{3}},
\end{gathered}
$$

where

$$
\lambda \equiv 2\left(\epsilon_{1}\left|C_{1}\right|^{2}+\epsilon_{1}\left|C_{2}\right|^{2}\right)
$$

$C_{1}$ and $C_{2}$ are constants such that $\left|C_{1}\right|^{2}+\left|C_{2}\right|^{2}=1$. The energy parameter $\lambda$ is necessarily positive because $\epsilon_{i}>0$ and the $\left|C_{i}\right|^{2}$ correspond to probabilities.

\section{Integrable Case: Absence of Coupling}

For the planar case, all $(a, P)$ that satisfy $\dot{a}=\dot{P}=0$ are equilibrium points. Each one is of the form $(\bar{a}, 0)$, where $\bar{a}$ satisfies

$$
\frac{\partial V}{\partial a}(\bar{a}, 0)=\frac{\lambda}{\bar{a}^{3}}
$$

The eigenvalues of the integrable system (8, 9) at the stationary point $(\bar{a}, 0)$ are

$$
\sigma= \pm \sqrt{-\frac{1}{M}\left(\frac{\partial^{2} V}{\partial a^{2}}(\bar{a}, 0)+\frac{3 \lambda}{\bar{a}^{4}}\right)}
$$

These eigenvalues are either real with opposite sign or are pure imaginary pairs, so in the linear analysis, each equilibrium is either a center or a saddle point. If

$$
A \equiv \frac{\partial^{2} V}{\partial a^{2}}(\bar{a}, 0)+\frac{3 \lambda}{\bar{a}^{4}}>0
$$


then every equilibrium point is a linear center. This holds, in particular, when the potential $V(a)$ has a single minimum (single-well potentials). Previous studies have focused on the harmonic potential

$$
V(a)=\frac{\mathcal{V}_{2}}{a_{0}^{2}}\left(a-a_{0}\right)^{2} \equiv V_{2}\left(a-a_{0}\right)^{2} .
$$

Another interesting single-well potential is the quartic potential

$$
V(a)=\frac{\mathcal{V}_{4}}{a_{0}^{4}}\left(a-a_{0}\right)^{4} \equiv V_{4}\left(a-a_{0}\right)^{4} .
$$

In the above equations, the $\mathcal{V}_{i}$ are dimensionless parameters.

It is insightful to examine the evolution (particularly in the chaotic case) of the quantum billiard system with the above two potentials and derive a mechanical anology in terms of spring stiffness. Given the same initial conditions and the quartic and quadratic potentials above (and assuming $V_{2}=V_{4}$ for ease for comparison), one observes that the phase-plane trajectory described by the evolution of $a$ and $P$ for the quartic potential (14) has a larger radius of curvature (that is, a smaller curvature). For all initial conditions, the trajectory in the quadratic potential has a larger maximum $a$. For initial conditions with sufficiently small $a(0)$, the quadratic potential induces trajectories with smaller maximum $|P|$, but the quartic potential eventually gives a larger maximum $|P|$ as $a(0)$ is increased.

An equilibrium for which $A<0$ is a saddle point. Since the present system is a Hamiltonian system, it is invariant under reflection about the $a$-axis, so that the eigenvectors representing the local stable and unstable manifolds are mapped to each other under this reflection. Since the only other possible types of equilibria are centers, it follows that if there is at least one saddle point, the system must have saddle connections. If there is 
exactly one, the connection is a homoclinic orbit, and there must be two of them emanating from the saddle because the system must have a center (in the right-half plane) on each side of the saddle.

As one considers increasingly excited states of the system (corresponding to larger quantum numbers), the energy parameter $\lambda$ is increased. Each saddle will eventually become a center as this occurs. The quantity $A$ vanishes at such an equilibrium point. The stationary point then has a double zero eigenvalue with the Jacobian

$$
\left(\begin{array}{ll}
0 & 1 \\
0 & 0
\end{array}\right)
$$

so that the saddle-center bifurcation that occurs has codimension two and gives rise to a global bifurcation corresponding to the breaking of the separatrix. [14, 11] To find the conditions satisfied at this point one can either solve the simultaneous equations $\dot{a}=0, \sigma=0$ or find the points at which the Hamiltonian has a double zero (which is equivalent to solving the system of equations $\left.H(\bar{a}, 0)=0, \frac{\partial H}{\partial a}(\bar{a}, 0)=0\right)$. If the potential has a constant term $V_{0}$, it does not change the evolution of the system since it does not appear in the equations of motion. We thus let $V_{0}=0$ without loss of generality. One finds that a saddle-center bifurcation occurs when

$$
\bar{\lambda}=\bar{a}^{3} \frac{\partial V}{\partial a}(\bar{a}, 0)
$$

at the point $(\bar{a}, 0)$ satisfying

$$
\frac{\partial V}{\partial a}(\bar{a}, 0)=-\frac{\bar{a}}{3} \frac{\partial^{2} V}{\partial a^{2}}(\bar{a}, 0) .
$$

Any solution to (17) giving $\lambda<0$ is discarded as nonsensical. 
At the saddle-center bifurcation point, the stable and unstable eigenvectors of the equilibrium coincide along the $a$-axis, so that the stable and unstable manifolds overlap near the stationary point. This cusp causes difficulties in numerical continuation attempts, as standard continuation techniques fail for this bifurcation. One observes that the two homoclinic orbits that exist when $A<0$ have coalesced into one. (As $A$ increases, the homoclinic orbit on the left shrinks, becoming a single point at the saddle-center. The orbit has infinite derivative with respect to arclength at the saddle-center point.)

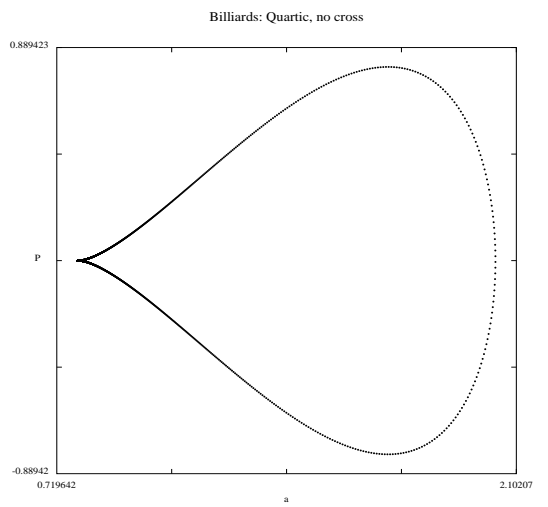

Figure 1: Approximate cuspidal homoclinic orbit. The initial point used was (0.7886751,0.001), just above the equilibrium.

As a specific example of this phenomenon, we consider the quartic potential

$$
V(a)=V_{4}\left(a-a_{0}\right)^{4}+V_{3}\left(a-a_{0}\right)^{3}+V_{2}\left(a-a_{0}\right)^{2}+V_{1}\left(a-a_{0}\right),
$$

where $V_{i} \equiv \frac{\mathcal{V}_{i}}{a_{0}^{i}}$. This potential has either one well or two. In the latter case, there is a single saddle-center bifurcation point. One can find $\bar{a}$ and 
$\bar{\lambda}$ exactly in this case, since the former is a root for a degree-three polynomial. We initially restrict ourselves to the case in which $V_{3}=V_{1}=0$, since all the dynamics of interest remain in this simpler case. Note that the potential is symmetric about $a_{0}$. For numerical purposes, consider the special case corresponding to the parameter values $a_{0}=1, V_{2}=-1$, and $V_{4}=1$. There is a saddle-center bifurcation at $\bar{\lambda}=\frac{1}{972}\left[3+\sqrt{3}^{3} \sqrt{3} \approx 0.1888176\right.$. The corresponding stationary point is $\left(\frac{1}{2}+\frac{\sqrt{3}}{6}, 0\right) \approx(0.7886751,0)$. Using DsTool [12], we plotted an approximation of the homoclinic orbit emanating from this equilibrium (Fig. 1).

More generally, one can consider even polynomials of higher degree in order to examine vibrating quantum billiards in an $N$-well potential. If the polynomial is of degree six or higher, one may not be able to solve for $\bar{a}$ exactly in terms of radicals by Galois theory [9], since equation (17) is polynomial of degree at least five. If its degree is exactly five, one can solve for $\bar{a}$ exactly in terms of elliptic functions. 223]

Consider the problem of starting at $\lambda<\bar{\lambda}$ and attempting to continue along the bifurcation curve past the saddle-center. Using AUTO[, \&] and the homotopy method encoded in HomCont, we followed the two homoclinic orbits for $\lambda=0.15$ (Fig. 2). The saddle connection in the present system has a codimension greater than one, as both regularity and non-degeneracy conditions are both violated. [5, 6] The present situation is degenerate because for all $\lambda<\bar{\lambda}$, there are two homoclinic orbits emanating from the saddle point. Regularity is violated because the saddle point's two eigenvalues are negatives of each other. (Moreover, their eigenvectors are related by reflection across the $a$-axis, since the system is Hamiltonian.) 


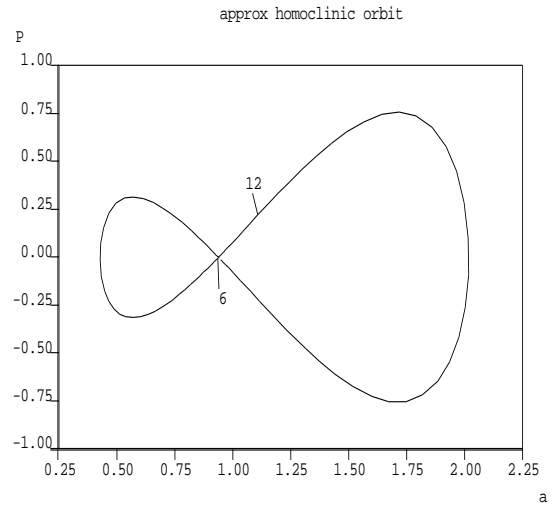

Figure 2: Homoclinic orbits emanating from $(0.8916637,0)$ for $\lambda=0.15$. The label 12 refers to the right homoclinic orbit, and the label 6 refers to the left one.

Because the present system is degenerate and irregular, one cannot continue (in $\lambda$ ) past the saddle-center directly, as described in the AUTO manual.[7] Hamiltonian systems possess a continua of homoclinic orbits, and there are numerical schemes that allow one to handle this phenomenon. One can exploit the reversibility of the system by computing only half of a given saddle connection. However, the cusp at the saddle-center point prevents this from working for the present system. AUTO is incapable of continuing past a cusp, because $\frac{\partial P}{\partial a}$ vanishes there and the pseudo-arclength step must become vanishingly small for such a continuation step to be successful. Because of machine precision, this cannot occur, and so one must "detune" the system to continue across the cusp.

In general, Hamiltonian systems are described by

$$
\dot{x}=J \nabla H(x, \lambda), x \in \mathbb{R}^{2 n},
$$


where

$$
J \equiv\left(\begin{array}{cc}
0 & I \\
-I & 0
\end{array}\right)
$$

is the canonical $2 n \times 2 n$ symplectic matrix [20]. One can detune the system by using a small perturbation parameter $\epsilon$ as follows. [5] The perturbed dynamical system,

$$
\dot{x}=J \nabla H(x, \lambda)+\epsilon \nabla H(x, \lambda)
$$

is no longer Hamiltonian, but the perturbation was constructed so that the locations of all equilibrium points are preserved. With this detuning, the saddle-center bifurcation becomes a saddle-node bifurcation (the eigenvalues of the stationary point are now of the form $a \pm \sqrt{b^{\prime}}(a \neq 0)$ rather than of the form $\pm \sqrt{b}$ ). One can then continue $\lambda$ past this point using AUTO (without utilizing the HomCont package). Using this technique, one can compute the value of $\lambda$ at which the saddle-center bifurcation occurs as well as the cusp point of the homoclinic orbit corresponding to that value. Moreover, once one has successfully continued past the cusp, one can simply let $\epsilon \longrightarrow 0$ and thereby work with the system when $\lambda>\bar{\lambda}$. This method of continuation is useful as long as one needs to get past the cusp rather than do computations at the cusp itself. For the present system, we used $\epsilon<0$, since in that case the equilibria that are centers for $\epsilon=0$ become stable spirals. The continuation curve (in $\lambda$ ) is shown in Figure 3 .

In general, AUTO has difficulties near cusps. As with DSTool, one can approximate the cuspidal homoclinic orbit using AUTO. In order to do this, one provides initial values for the HomCont continuation parameters (corresponding to the initial point in the $(a, P)$-plane) to the right of the saddle 


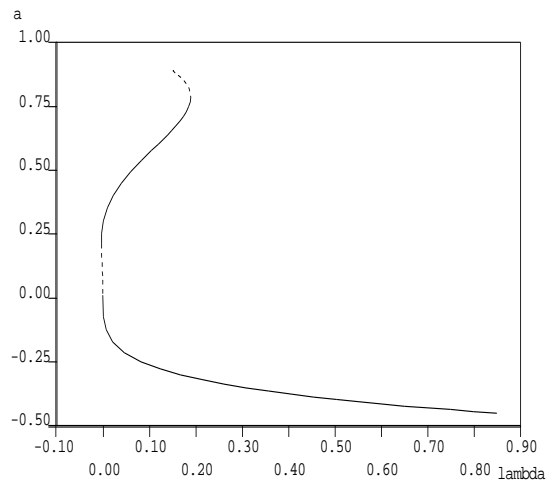

Figure 3: Continuation of the detuned system in the parameter $\lambda$.

point $\left(a^{*}, 0\right)$. This allows AUTO to continue along the homoclinic orbit for values of $\lambda$ closer to $\bar{\lambda}$ than if one had started as close to the saddle point as machine precision would allow. For the present example, the closest accurate plot we obtained was for $\lambda=0.1887$. The right homoclinic orbit is pictured in Figure 1 and the left one is pictured in Figure 5. Observe that the one on the right looks like it has a cusp at the saddle point and that the left one is very small. As the saddle-center is approached, the left homoclinic orbit shrinks to a single point and the right one becomes more cusplike.

\section{Chaotic Case: Presence of Coupling}

There are other methods one can use to perform analysis near the cusp. One can, for example, use shooting methods. 10 (AUTO uses a collocation method.) Saddle-centers are a degenerate case of Takens-Bogdanov (TB) bifurcations [11], so one can add dummy parameters and analyze the cuspidal loop by computing the locus of a TB bifurcation in parameter space, moving along the TB curve until one finds a Hopf bifurcation of another equilibrium, 


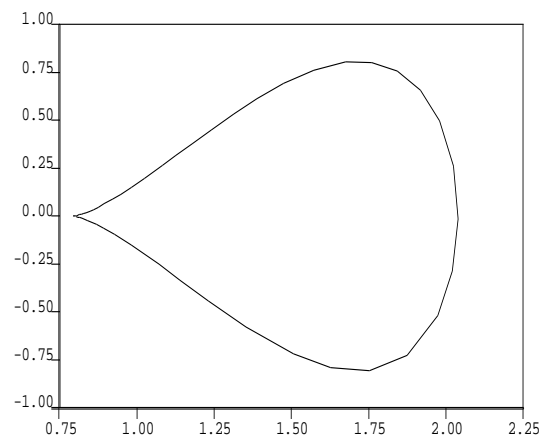

Figure 4: Right homoclinic orbit for $\lambda=0.1887$.

and then following the evolution of the periodic orbit created in the Hopf bifurcation as the parameters follow the TB bifurcation curve. If a cuspidal loop exists, this method will find it when the periodic orbit collapses into the cusp point.

For the chaotic case, the equilibrium points satisfy $x=P=y=0$, $z= \pm 1$, and $\frac{\partial V}{\partial a}=\frac{2}{a^{3}}\left(\epsilon_{+} \pm \epsilon_{-}\right)$, where the factor of \pm 1 in the last quantity corresponds to the equilibrium value of $z$. The eigenvalues for the equilibria of the chaotic system (Eqs. 2 6) are of the form

$$
\sigma=0, \pm \frac{\sqrt{-2 M(\alpha \pm \sqrt{\beta})}}{2 M a^{2}}
$$

where both $\alpha$ and $\beta$ have terms whose signs depend on whether $z$ is 1 or -1 . The quantities $\alpha_{ \pm}$and $\beta_{ \pm}$are given by

$$
\begin{gathered}
\alpha_{+}=a^{4} \frac{\partial^{2} V}{\partial a^{2}}+\omega_{0}^{2} M-4 \mu^{2} \epsilon_{-}+6\left(\epsilon_{+}+\epsilon_{-}\right), \\
\alpha_{-}=a^{4} \frac{\partial^{2} V}{\partial a^{2}}+\omega_{0}^{2} M+4 \mu^{2} \epsilon_{-}+6\left(\epsilon_{+}-\epsilon_{-}\right), \\
\beta_{+}=I_{1}+I_{2}^{+}+I_{3}^{+}+I_{4}^{+},
\end{gathered}
$$


where

$$
\begin{gathered}
I_{1} \equiv a^{8}\left(\frac{\partial^{2} V}{\partial a^{2}}\right)^{2} \\
I_{2}^{+} \equiv \frac{\partial^{2} V}{\partial a^{2}}\left(12 a^{4} \epsilon_{+}-2 \omega_{0}^{2} M a^{4}+12 a^{4} \epsilon_{-}-8 \mu^{2} a^{4} \epsilon_{-}\right) \\
I_{3}^{+} \equiv 16 \mu^{4} \epsilon_{-}^{2}-8 \omega_{0}^{2} M \mu^{2}-48 \mu^{2} \epsilon_{-}^{2}-12 \omega_{0}^{2} M \epsilon_{+}+72 \epsilon_{+} \epsilon_{-}-12 \omega_{0}^{2} M \epsilon_{-} \\
I_{4}^{+} \equiv-48 \mu^{2} \epsilon_{+} \epsilon_{-}+\omega_{0}^{4} M^{2}+36\left(\epsilon_{+}^{2}+\epsilon_{-}^{2}\right)
\end{gathered}
$$

and

$$
\beta_{-}=I_{1}+I_{2}^{-}+I_{3}^{-}+I_{4}^{-}
$$

where $I_{1}$ is as before,

$$
\begin{gathered}
I_{2}^{-} \equiv \frac{\partial^{2} V}{\partial a^{2}}\left(12 a^{4} \epsilon_{+}-2 \omega_{0}^{2} M a^{4}-12 a^{4} \epsilon_{-}+8 \mu^{2} a^{4} \epsilon_{-}\right) \\
I_{3}^{-} \equiv 16 \mu^{4} \epsilon_{-}^{2}+8 \omega_{0}^{2} M \mu^{2}-48 \mu^{2} \epsilon_{-}^{2}-12 \omega_{0}^{2} M \epsilon_{+}-72 \epsilon_{+} \epsilon_{-}+12 \omega_{0}^{2} M \epsilon_{-} \\
I_{4}^{-} \equiv 48 \mu^{2} \epsilon_{+} \epsilon_{-}+\omega_{0}^{4} M^{2}+36\left(\epsilon_{+}^{2}+\epsilon_{-}^{2}\right) .
\end{gathered}
$$

Analogous to the planar system, only a generalization of saddle-center bifurcations can occur. As the energy is increased, a bifurcation corresponds to an increase in the dimension of the center manifold by two as a pair of real eigenvalues of opposite signs becomes a pair of pure imaginary eigenvalues. For a double-well potential, the only possibility is a jump in the dimension of the center manifold from three to five. For more complicated potentials, there may be parameter values with a one-dimensional center manifold. As before, one can determine the location of this bifurcation by finding the equilibria for which $H(a, P)$ has a double root. One again finds that the equilibrium point $(0,0, \pm 1, \bar{a}, 0)$ at the bifurcation satisfies

$$
\frac{\partial V}{\partial a}(\bar{a}, 0)=-\frac{\bar{a}}{3} \frac{\partial^{2} V}{\partial a^{2}}(\bar{a}, 0) .
$$




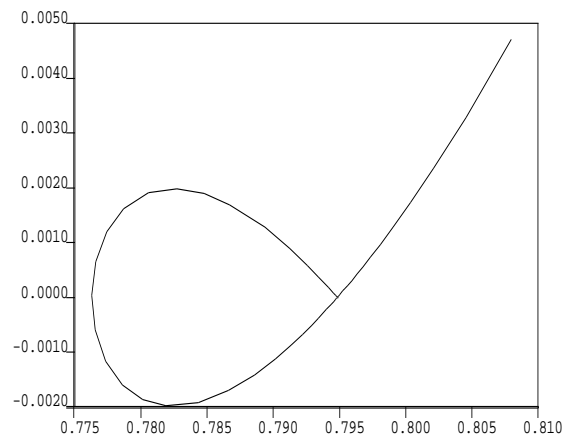

Figure 5: Left homoclinic orbit for $\lambda=0.1887$.

Finding the parameter values at which this occurs is not the simple issue it was in the previous case. In the planar case, $\lambda$ was a probabilistic weighting of two energies, so it could be varied continuously past the bifurcating value $\bar{\lambda}$. However, the parameters $\epsilon_{1}, \epsilon_{2}$ in the present system are part of a discrete energy spectrum, and so one cannot vary them continuously. In practice, therefore, this model predicts superposition states on each side of the bifurcation, but one does not expect to observe the system at a bifurcating value of $\left(\epsilon_{1}, \epsilon_{2}\right)$ because the set of all $\left(\epsilon_{1}, \epsilon_{2}\right)$ that correspond to bifurcating values has measure zero. (One could vary $V_{2}$ continuously if one wanted to examine bifurcations corresponding to a change in the quartic potential. If $V_{2}$ is negative and sufficiently small for a given $V_{4}$ or if it's positive, the system will not exhibit a saddle-center.) Additionally, numerical observations indicate that the bifurcation occurs at low energies (corresponding to superpsoitions of low quantum number states), so that for a given billiard system, most superposition states will exhibit an evolution with a five-dimensional center manifold. The bifurcation under study may thus occur as one considers superpositions of increasingly excited states of the quantum billiard. 
Numerical investigations have shown that homoclinic orbits must exist for this five-dimensional system (Fig. 6). Slight perturbations away from the homoclinic orbits lead to homoclinic tangles (Fig. 7), which are traditionally analyzed using symbolic dynamics. The details have not been worked out, but the existence of a homoclinic orbit for the present case can be shown analytically as follows. There is only one saddle point, so any saddle connection would have to be a homoclinic connection. By Hamiltonian symmetry and the existence of a center to the right of the "saddle-like" (in the sense that it has one-dimensional stable and unstable manifolds) equilibrium, there must exist a structure to its right that looks like a homoclinic orbit when projected into the $(a, P)$-plane. It may not be a homoclinic orbit, because one must consider the value of $(x, y, z)$ at the point $(\tilde{a}, 0)$ where the projection intersects the $a$-axis. One thereby considers the 2 -sphere $S^{2}$ and two trajectories on it that start at the same point. One curve begins at $t=\infty$ from the stable manifold, and the other starts at $t=-\infty$ from the unstable manifold. One looks at the intersection of one trajectory at time $t$ with the other at time $-t$. If one can prove that such a point exists at a time $T$, then repeating the argument shows that such an intersection occurs at infinitely many times. To complete the proof, one must show that $P=0$ at one of these points.

Note that the homoclinic orbit for the chaotic system (Eqs. 20 6) emanates and terminates from a nonhyperbolic equilibrium, which increases the difficulty of numerical studies. [5, 6] As with the planar system discussed earlier, there is also a cusplike structure as the stable and unstable manifolds coalesce along the $a$-axis.

As an anology, consider two undamped springs, one with a linear restoring 


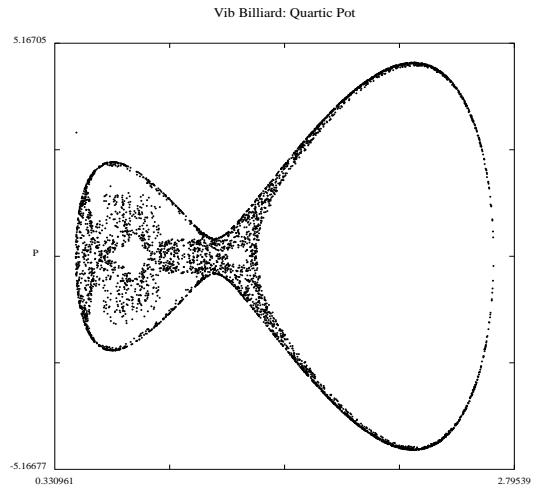

Figure 6: Poincaré section projected into the $(a, P)$-plane demonstrating that there must exist a homoclinic orbit.

force $\left(F_{1}=-k x\right)$ and a stiffer spring with a cubic one $\left(F_{3}=-k x^{3}\right)$. These two springs (with mass $m=1$ ) are described, respectively, by the differential equations

$$
\ddot{x}+k x=0,
$$

and

$$
\ddot{x}+k x^{3}=0 .
$$

One observes the same stiffness phenomena for these spring systems as we did for the integrable case of the vibrating billiard. (For example, the phase space trajectory of the cubic spring (Eq. 31) has a larger radius of curvative than the analogous one in the linear spring system. There is a correspondence with the other properties we discussed as well.) By analogy with mass-spring systems, we thus conclude that it is reasonable to consider the "stiffness" of the potential in which a quantum billiard resides as an object of interest. 


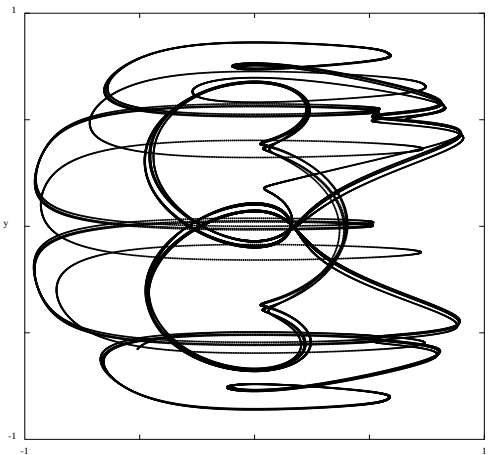

Figure 7: A homoclinic tangle projected onto the $(x, y)$-plane of the Bloch sphere.

The analogy with spring stiffness carries through in the chaotic case as well as in the planar case. For the same initial conditions, we consider the potentials

$$
V(a)=V_{2}\left(a-a_{0}\right)^{2}
$$

and

$$
V(a)=V_{4}\left(a-a_{0}\right)^{4}
$$

where for ease of comparison, $V_{2}=V_{4}$. As shown in Fig. 8, the quartic potential gives trajectories with a larger radius of curvature than those in the quadratic potential. It thus makes sense to consider a potential's stiffness in the chaotic case as well as in the integrable one. 


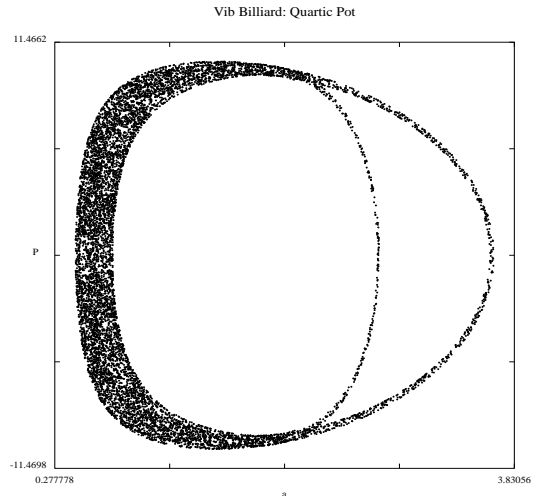

Figure 8: Chaotic Poincaré maps in the $(a, P)$-plane for billiards in both a quartic and a quadratic potential. The plot from the quartic potential is the one with a smaller maximum value for the distance $a$. Notice that the trajectory corresponding to the quartic potential generically has a larger radius of curvature.

\section{Conclusions}

We showed that saddle-centers are the only type of bifurcations that can occur in one dov quantum billiards. When there is no coupling in the superposition state, we showed how to analyze the resulting planar system analytically and numerically. Considering a double-well potential as a representative example, we demonstrated how to locate bifurcations in parameter space. We also discussed how to approximate the cuspidal loop using AUTO as well as how to continue past it by detuning the dynamical system. We also mentioned a shooting method for a more detailed analysis of the cuspidal loop. Moreover, we showed that when there is coupling, the 
resulting five-dimensional system - though chaotic - has a similar underlying structure. We verified numerically that both homoclinic orbits and cusps occur. Small perturbations of the system reveal homoclinic tangles that typify chaotic behavior.

\section{Acknowledgements}

We would like to thank Joel Ariaratnam, Richard Casey, John Guckenheimer, and Steven Wirkus for useful discussions concerning this project. Additionally, we express our gratitude toward Alan Champneys for several productive suggestions regarding the numerics, Alejandro Rodríguez-Luis for providing a preprint of his manuscript, and Paul Steen, whose guidance for this work as a project for $\mathrm{ChE} 753$ (on which this paper is based) was particular valuable.

\section{References}

[1] L. Allen and J. H. Eberly. Optical Resonance and Two-Level Atoms. Dover Publications, Inc., New York, NY, 1987.

[2] R. Badrinarayanan and J. V. José. Spectral properties of a Fermi accelerating disk. Physica D, 83:1-29, 1995.

[3] R. Blümel and B. Esser. Quantum chaos in the Born-Oppenheimer approximation. Physical Review Letters, 72(23):3658-3661, 1994.

[4] R. Blümel and W. P. Reinhardt. Chaos in Atomic Physics. Cambridge University Press, Cambridge, England, 1997. 
[5] Alan R. Champneys, Yu. A. Kuznetsov, and Björn Sandstede. A numerical toolbox for homoclinic bifurcation analysis. International Journal of Bifurcation and Chaos, 6(5):867-887, 1996.

[6] Alan R. Champneys and Yuri A. Kuznetsov. Numerical detection and continuation of codimension-two homoclinic bifurcations. International Journal of Bifurcation and Chaos, 4(4):785-822, 1994.

[7] Eusebius J. Doedel, Alan R. Champneys, Thomas F. Fairgrieve, Yuri A. Kuznetsov, Björn Sandstede, and Xianjun Wang. AUTO 97: Continuation and Bifurcation Software for Ordinary Differential Equations (with Hom Cont), March 1998.

[8] Eusebius J. Doedel, Mark J. Friedman, and Anand C. Monteiro. On locating homoclinic and heteroclinic orbits. Technical report, Center for Applied Mathematics, Cornell University, June 1993.

[9] David S. Dummit and Richard M. Foote. Abstract Algebra. Prentice Hall, Englewood Cliffs, NJ, 1991.

[10] E. Freire, L. Pizarro, and Alejandro J. Rodríguez-Luis. Numerical continuation of homoclinic orbits to non-hyperbolic equilibria in planar systems. Preprint, 1999.

[11] John Guckenheimer and Philip Holmes. Nonlinear Oscillations, Dynamical Systems, and Bifurcations of Vector Fields. Number 42 in Applied Mathematical Sciences. Springer-Verlag, New York, NY, 1983.

[12] John Guckenheimer, B. A. Meloon, M. R. Myers, F. J. Wicklin, and P. A. Worfolk. DsTool: A Dynamical Systems Toolkit with an Interactive 
Graphical Interface: User's Manual. Center for Applied Mathematics, Cornell University, Ithaca, NY, December 1997. Version Tk Draft.

[13] Martin C. Gutzwiller. Chaos in Classical and Quantum Mechanics. Number 1 in Interdisciplinary Applied Mathematics. Springer-Verlag, New York, NY, 1990.

[14] Jack Hale and Hüseyin Koçak. Dynamics and Bifurcation. Number 3 in Texts in Applied Mathematics. Springer-Verlag, New York, NY, 1991.

[15] Anatole Katok and Boris Hasselblatt. Introduction to the Modern Theory of Dynamical Systems. Cambridge University Press, New York, NY, 1995.

[16] Richard L. Liboff. Introductory Quantum Mechanics. Addison-Wesley, San Francisco, CA, 3rd edition, 1998.

[17] Richard L. Liboff and Mason A. Porter. Quantum chaos for the radially vibrating spherical billiard. Chaos, 10(2):366-370, 2000.

[18] J Lucan. Quantum Dots. Springer, New York, NY, 1998.

[19] S. W. MacDonald and A. N. Kaufman. Wave chaos in the stadium: Statistical properties of short-wave solutions of the Helmholtz equation. Physical Review A, 37:3067, 1988.

[20] Jerrold E. Marsden and Tudor S. Ratiu. Introduction to Mechanics and Symmetry. Number 17 in Texts in Applied Mathematics. SpringerVerlag, New York, NY, second edition, 1999. 
[21] Mason A. Porter and Richard L. Liboff. Vibrating quantum billiards on Riemannian manifolds. International Journal of Bifurcation and Chaos, To appear September, 2001.

[22] Roger Temam. Infinite-Dimensional Dynamical Systems in Mechanics and Physics. Number 68 in Applied Mathematical Sciences. SpringerVerlag, New York, NY, 2nd edition, 1997.

[23] Stephen Wolfram. Solving the quintic with Mathematica. http://library.wolfram.com/examples/quintic/, 1999.

[24] S Wong. Introductory Nuclear Physics. Prentice Hall, Englewood Cliffs, NJ, 1990.

[25] H Zaren, K Vahala, and A Yariv. Gain spectra of quantum wires with inhomogeneous broadening. IEEE Journal of Quantum Electronics, 25:705, 1989.

\section{Figure Captions}

Figure 1: Approximate cuspidal homoclinic orbit. The initial point used was (0.7886751,0.001), just above the equilibrium.

Figure 2: Homoclinic orbits emanating from $(0.8916637,0)$ for $\lambda=0.15$. The label 12 refers to the right homoclinic orbit, and the label 6 refers to the left one. 
Figure 3:Continuation of the detuned system in the parameter $\lambda$.

Figure 4: Right homoclinic orbit for $\lambda=0.1887$.

Figure 5: Left homoclinic orbit for $\lambda=0.1887$.

Figure 6: Poincaré section projected into the $(a, P)$-plane demonstrating that there must exist a homoclinic orbit.

Figure 7: A homoclinic tangle projected onto the $(x, y)$-plane of the Bloch sphere.

Figure 8: Chaotic Poincaré maps in the $(a, P)$-plane for billiards in both a quartic and a quadratic potential. The plot from the quartic potential is the one with a smaller maximum value for the distance $a$. Notice that the trajectory corresponding to the quartic potential generically has a larger radius of curvature. 